\title{
MULTIPLIERS ON WEIGHTED HARDY SPACES OVER CERTAIN TOTALLY DISCONNECTED GROUPS
}

\author{
TOSHIYUKI KITADA \\ Department of Mathematics \\ Faculty of General Education \\ Hi rosaki University \\ Hi rosaki 036, JAPAN \\ (Received June 24, 1987 and in revised form October 19, 1987)
}

ABSTRACT. In this note, we consider the multipliers on weighted $\mathrm{H}^{1}$ spaces over totally disconnected locally compact abelian groups with a suitable sequence of open compact subgroups (Vilenkin groups). We first show an $\left(H^{1}, L^{l}\right)$ multiplier result from which Onneweer's theorem follows. We also give an $\left(\mathrm{H}^{1}, \mathrm{H}^{1}\right)$ multiplier result under a condition of Baernstein-Sawyer type.

KEY WORDS AND PHRASES. Totally disconnected groups, Weighted $\mathrm{H}^{1}$ spaces, Weighted $L^{\mathrm{p}}$ spaces, Multipliers.

1980 AMS SUBJECT CLASSIFICATION CODE. 43A22, $43 \mathrm{~A} 70$.

1. INTROUDCTION.

Recently, Onneweer obtained a weighted $\mathrm{L}^{\mathrm{p}}$ multiplier theorem [1, Theorem 1] over a Vilenkin group which is a generalization of Taibleson's theorem over a local field.

In this note, we show a weighted $\left(\mathrm{H}^{1}, \mathrm{~L}^{1}\right)$ multipler theorem under a weaker hypothesis than [1, Proposition 2], and show the Onneweer's theorem, by using an extended interpolation theorem for weighted $\mathrm{H}^{1}$ and $\mathrm{L}^{\mathrm{p}}$ spaces. We do not know whether this multiplier is also a weighted $\left(\mathrm{H}^{1}, \mathrm{H}^{1}\right)$ multiplier. But we are able to show that a Baernstein-Sawyer type condition [2] which is stronger than Onneweer's, implies a weighted $\left(\mathrm{H}^{1}, \mathrm{H}^{1}\right)$ result. This is also a generalization of Theorem 2 [2].

2. DEFINITIONS AND NOTATIONS.

Throughout this note, G will denote a locally compact abelian group with a sequence $\left\{G_{n}\right\}_{-\infty}^{\infty}$ such that

(i) each $G_{n}$ is an open compact subgroup of $G$,

(11) $G_{n+1} \subsetneq G_{n}$ and order $\left(G_{n} / G_{n+1}\right)<\infty$,

(iii) $\bigcup_{\infty}^{\infty} G_{n}=G$ and $\bigcap_{\infty}^{\infty} G_{n}=\{0\}$. 
Moreover we shall assume that $G$ is order-bounded, 1.e.;

$$
B:=\sup \left\{\text { order }\left(G_{n} / G_{n+1}\right) ; n \in Z\right\}<\infty \text {. }
$$

Let $\Gamma$ denote that dual group of $G$ and for each $n \in z$, let $\Gamma_{n}$ denote the annihilator of $G_{n}$. Then we have

$$
\begin{aligned}
& \text { (1)' each } \Gamma_{n} \text { is an open compact subgroup of } \Gamma \text {, } \\
& \text { (ii)' } \Gamma_{n} \subset_{n+1} \text { and order }\left(\Gamma_{n+1} / \Gamma_{n}\right)=\operatorname{order}\left(G_{n} / G_{n+1}\right) \text {, } \\
& \text { (iii) } \bigcup_{-\infty}^{\infty} \Gamma_{n}=\Gamma \text { and } \bigcap_{\infty}^{\infty} \Gamma_{n}=\{1\} \text {. }
\end{aligned}
$$

We choose Haar measures $\mu$ on $G$ and $\lambda$ on $\Gamma$ so that $\mu\left(G_{0}\right)=\lambda\left(\Gamma_{0}\right)=1$, then $\mu\left(G_{n}\right)=\left(\lambda\left(\Gamma_{n}\right)\right)^{-1}:=\left(m_{n}\right)^{-1}$ for each $n \in Z$. For an arbitrary set $A$ we denote its indicator function by $\xi_{A^{*}}$ The symbols $\wedge$ and $\vee$ will be used to denote the Fourier and inverse Fourier transform respectively. It is easy to see that for each $n \in Z$ we have $\left(\xi_{G_{n}}\right)^{\hat{n}}=\left(\lambda\left(\Gamma_{n}\right)\right)^{-1} \xi_{\Gamma_{n}}$. We set $D_{n}:=\left(\mu\left(G_{n}\right)\right)^{-1} \xi_{G_{n}}$ for each $n \in Z$.

We now define the weighted $L^{p}$ spaces. For $\alpha \in R$, we define the function $v_{\alpha}$ on $G$ by $v_{\alpha}(x)=\left(m_{n}\right)^{-\alpha}$ if $x \in G_{n} \backslash G_{n+1}(n \in Z) ;=0$ if $x=0$. We denote the $L^{p}$ spaces with respect to the measure $d \mu_{\alpha}:=v_{\alpha} d \mu$ on $G$ by $L_{\alpha}^{P}(G)$, simply $L_{\alpha}^{p}$. Also for $1<p<\infty$, we set

$$
\|f\|_{p, \alpha}:=\left(\int_{G}|f(x)|^{P} d \mu_{\alpha}\right)^{1 / p}
$$

Let $S(G)$ be the set of all functions $\phi$ on $G$ such that $\phi$ has compact support and is constant on the cosets of some subgroup $G_{n}$ ( $n$ depends on $\phi$ ) of $G$. The functions in $S(G)$ are called test functions on $G$. It is well known that if $\alpha>-1$, then $S(G)$ is dense in $\mathrm{L}_{\alpha}^{\mathrm{p}}$ for $1<\mathrm{p}<\infty$.

In order to define the weighted Hardy spaces on $G$, we first define weighted atoms on $G$. Let $1<q<\infty$. A function $a(x)$ on $G$ is $a(1, q)_{\alpha}$ atom if there exists an interval $I=I_{n}(x):=x+G_{n}, x \in G, n \in Z$ such that

(1) supp a is contained in $I$,

$$
\begin{aligned}
\left(\frac{1}{\mu_{\alpha}(I)} \int_{I}|a(x)|^{q} d \mu_{\alpha}\right)^{1 / q}<\mu_{\alpha}(I)^{-1}, \text { if } 1<q<\infty \\
\text { and }|a(x)|<\mu_{\alpha}(I)^{-1} \text {, if } q=\infty,
\end{aligned}
$$

(iii) $\int a(x) d \mu=0$.

The weighted Hardy $\operatorname{space}_{\infty} H_{\alpha}^{1, q}(G), \operatorname{simply} H_{\alpha}^{1, q}$, is the space of all functions $f$ on on $G$ such that $f(x)=\sum_{0}^{\infty} \lambda_{k} a_{k}(x)$,

where the $a_{k}{ }^{\prime} s$ are $(1, q)_{\alpha}$ atoms and $\Sigma_{0}^{\infty}\left|\lambda_{k}\right|<\infty^{\infty}$. We set ||$f\left|{ }_{H_{\alpha}, q}:=\operatorname{lnf} \Sigma_{0}^{\infty}\right| \lambda_{k} \mid$, where the infimum is taken over all such decompositions. Then $H_{\alpha}^{1, q}$ is a subspace of $L_{\alpha}^{1}$ and 

a Banach space with the norm $\|\cdot\|_{H_{\alpha}} \|^{\circ}$ It also follows easily from the definition
that

$$
H_{\alpha}^{1, \infty} \subset{ }_{\alpha}^{1, q_{2}} \subset{ }^{H_{\alpha}^{1, q_{1}}}
$$

whenever $1<q_{1}<q_{2}<\infty$. We denote $H_{\alpha}^{1, \infty}$ by $H_{\alpha}^{1}$. In the following section, we show that $H_{\alpha}^{1, q}=H_{\alpha}^{1}$ if $-1<\alpha<0$ and $1<q<\infty$.

We say that $m \in L^{\infty}(\Gamma)$ is an $(X, Y)$ multiplier (or a multiplier on $X$, when $X=Y$ ) if there exists a constant $C>0$ so that

$$
\left\|(m \hat{\phi})^{v}\right\|_{Y}<c\|\phi\|_{X} \quad \text { for all } \phi \in X \cap s(G)
$$

where $X$ and $Y$ are equal to $H_{\alpha}^{1}$ or $L_{\alpha}^{p}$.

According to $[1]$, we say that $\phi \in L^{\infty}(\Gamma)$ satisfies condition $C(k, r)$ for some $k \in Z$ and $r \in[1, \infty)$ if there exist $C, \varepsilon>0$ so that for all $\ell, n \in Z$ with $\mathrm{n}<\ell$ have

$$
\begin{aligned}
& \sup \left\{\left(\int_{G_{n} \backslash G_{n+1}}\left|\left(\phi^{k}\right)^{v}(x-y)-\left(\phi^{k}\right)^{\vee}(x)\right|^{r} d \mu\right)^{1 / r} ; y \in G_{\ell}\right. \\
& <C\left(m_{n}\right)^{1 / r^{\prime}+\varepsilon}\left(m_{\ell}\right)^{-\varepsilon} \text {, if } 1<r<\infty,
\end{aligned}
$$

and there exists $C>0$ so that for all $\ell \in Z$ we have

$$
\sup \left\{\int_{G G_{\ell}}\left|\left(\phi^{k}\right)^{v}(x-y)-\left(\phi^{k}\right)^{v}(x)\right| d \mu ; y \quad G_{\ell}\right\}<c \text {, if } r=1 \text {, }
$$

where $\phi^{k}=\phi \xi_{\Gamma_{k}}$ for each $k \in Z$ and $r^{\prime}$ denotes the conjugate exponent of $r$.

Let $-\infty<\alpha<\infty, 1<p<\infty$ and $0<q<\infty$. A function $f$ on $G$ belongs to the Herz s pace $k_{p}^{\alpha, q}(G)$, simply $k_{p}^{\alpha, q}$, if

$$
\|f\|_{K_{p}^{\alpha, q}}:=\left(\sum_{-\infty}^{\infty}\left\|\left(m_{n}\right)^{-\alpha} f \underset{G_{n}}{\xi}\right\|_{n+1} \| p\right)^{1 / q}<\infty,
$$

with the usual modification if $q=\infty[3]$.

We now state the main theorems:

THEOREM 1. Let $\phi \in L^{\infty}(\Gamma)$ and suppose that $\phi$ satisfies condition $C(k, r)$ for some $k \in Z$ and $r \in[1, \infty)$. Then $\phi^{k}$ is an $\left(H_{\alpha}^{1}, L_{\alpha}^{1}\right)$ multiplier for $-1 / r^{\prime}<\alpha<0$.

As a Corollary we obtain Theorem 1 of [1]:

COROLLARY. Let $\phi \in L^{\infty}(\Gamma)$. (i) Suppose that condition $C(k, r)$ holds for all $k \in z$, for some $r \in(1, \infty)$, and with constants $C$ and $\varepsilon$ independent of $k \in z$. If $\phi$ is a multiplier on $\mathrm{L}_{\alpha_{0}}^{2}$ for some $\alpha_{0}$ with $-1 / \mathrm{r}^{\prime}<\alpha_{0}<1 / \mathrm{r}^{\prime}$, then $\phi$ is a multiplier on $\mathrm{L}_{\alpha}^{\mathrm{p}}$ for al1 $\mathrm{p}, \alpha$ such that $1<\mathrm{p}<\infty$ and $-\left|\alpha_{0}\right|<\alpha<(\mathrm{p}-1)\left|\alpha_{0}\right|$.

(1i) If $C(k, 1)$ holds for all $k \in Z$, and with $C$ independent of $k \in Z$, then $\phi$ is a multiplier on $\mathrm{L}^{\mathrm{p}}$ for $1<\mathrm{p}<\infty$. 
THEOREM 2. Let $\phi \in \mathrm{L}^{\infty}(\Gamma)$ and suppose that there exist $r \in[1, \infty)$ and $\varepsilon>0$ such that

$$
\left\|\left(\phi_{j}\right)^{v}\right\| k_{r}^{\varepsilon+1 / r^{\prime}, \infty}<c\left(m_{j}\right)^{-\varepsilon} \text { for all } j \in z
$$
$\begin{aligned} \text { where } \phi_{j}: & =\phi \xi_{\Gamma_{j+1}} \backslash \Gamma_{j} \text { for each } j \in \mathrm{z} \text {, then } \phi \text { is a multiplier on } H_{\alpha}^{1} \text { for } \\ -1<r^{\prime} \alpha & <0 .\end{aligned}$

3. PRELIMINARY RESULTS.

To prove Theorem 2, we need the "maximal function" characterization of $H_{\alpha \text {. For }}^{1}$ $f$ locally in $L_{\alpha}^{1}(G)$ we define the maximal function $M_{\alpha} f$ of $f$ by

$$
M_{\alpha} f(x):=\sup _{I}\left\{\frac{1}{\mu_{\alpha}(I)} \int_{I}|f(y)| d \mu_{\alpha}(y)\right\}
$$

where the I's are intervals containing $x$. When $\alpha=0$, we denote $M_{\alpha}$ by $M$, simply.

LEMMA. Let $\alpha>-1$.

(b) $\quad M_{\alpha}$ is of weak-type (1.1) on $L_{\alpha}^{1}$ and is of type $(p, p)$ on $L_{\alpha}^{p}$ for $1<p<\infty$,

(c) If $\alpha<0$, then for all interval I

$$
\mu_{\alpha}(I)<C \mu(I) \inf \left\{v_{\alpha}(y) ; y \in I, y \neq 0\right\}
$$

(d) If $\alpha<0$, then $M$ is of weak-type $(1,1)$ on $\mathrm{L}_{\alpha}^{1}$.

PROOF. (a) and (c) are Lemmas 1(b) and (c) in [1]. (b) follows from (a). By (c), we have that $\operatorname{Mf}(x)<C M_{\alpha} f(x)$ for each $x \in G$. Then (d) follows from (b). THEOREM A. Let $\alpha>-1$. An $\mathrm{f} \mathrm{L}_{\alpha}^{1}$ belongs to $\mathrm{H}_{\alpha}^{1}$ if and only if $\mathrm{f}^{*}:=\mathrm{Mf}_{\alpha} \mathrm{L}_{\alpha}^{1}$. Moreover $\|\mathrm{f}\|_{\mathrm{H}_{\alpha}}$ is equivalent to $\|\mathrm{f*}\|_{1, \alpha}$.

A slight modification of the argument in [2] establishes the result, so we omit the proof.

THEOREM B. Let $-1<\alpha<0$. Then $H_{\alpha}^{1, q} \simeq H_{\alpha}^{1}$, for $1<q<\infty$.

PROOF. We have already seen that $H_{\alpha}^{1}$ is continuously included in $H_{\alpha}^{1, q}$, for each $1<q<\infty$. In order to establish the opposite inclusion, it suffices to show that a $(1, q)_{\alpha}$ atom a has the representation

$$
a(x)=\sum_{0}^{\infty} \quad \lambda_{j} a_{j}(x)
$$

where each $a_{j}$ is $a(1, \infty)_{\alpha}$ atom and $\sum_{0}^{\infty}\left|\lambda_{j}\right|<c, c$ independent of a. Like the nonweighted case, this can be done by using the Calderon-Zygmund decomposition [4], [5].

Let a be a $(1, q)_{\alpha}$ atom that is suppported on $I:=x_{0}+G_{n_{0}}\left(x_{0} \in G, n_{0} \in Z\right)$. We let $b(x):=\mu_{\alpha}(I) a(x)$, then supp $b \subset I, \int b(x) d \mu(x)=0$, and $\|\left. b\right|_{q, \alpha} ^{q} \leqslant \mu_{\alpha}(I)$. 
For $t>0$ (we shall be explicit later), we denote the open set

$\left\{x \in G: M_{\alpha}^{q}(b)>t\right\}:=\left\{x \in G ; M_{\alpha}\left(|b|^{q}\right)(x)>t^{q}\right\}$ by $U_{t}$. We note that $U_{t} \subset$ I for $t$ $>$ 1. (This is easily seen from the fact that for any two intervals in $G$, they are disjoint or one contains the other). Lemma (b) implies that

$$
\mu_{\alpha}\left(U_{t}\right)<c|| b||_{q, \alpha}^{q} / t^{q}<C \mu_{\alpha}(I) / t^{q}
$$

and $H_{\alpha}\left(G_{k}\right) \rightarrow \infty$ as $k \rightarrow-\infty[1$, Lemma (a)]. Thus we have the decomposition

$U_{t}:=\bigcup_{j} I_{j}$; where the $I_{j}$ 's are naximal disjoint sub-intervals of $U_{t}$. The CalderonZygmund decomposition is now that $b(x)=g_{0}(x)+\sum_{j}$, where $g_{0}(x)=b(x)$ if

$$
x \notin U_{t} ;=m\left(b, I_{j}\right) \text { if } x \in I_{j} \text { and } h_{j}(x)=\left(b(x)-g_{0}(x)\right) \xi_{I_{j}}(x) \text {, and where } m\left(b, I_{j}\right)
$$

denotes the average of $b$ over $I_{j}$ with respect to $\mu$. Then the maximality of

the $I_{j}$ 's and Lemma (a), (b) imply that $\left|g_{0}(x)\right|<C_{0} t, \mu_{\alpha}$-a.e. and

$$
\frac{1}{\mu_{\alpha}\left(I_{j}\right)} \int_{I_{j}}\left|h_{j}\right| d \mu_{\alpha} \leqslant\left(\frac{1}{\mu_{\alpha}\left(I_{j}\right)} \int_{I_{j}}\left|h_{j}\right|^{q} d \mu_{\alpha}\right)^{1 / q}<2 C C_{0} t:=c_{1} t
$$

by Lemma (c). If we set $\left(C_{1} t\right)^{-1} h_{j}=b_{j}$, then $b_{j}$ is supported in $I_{j}, \int b_{j} d \mu=0$ and $\left\|b_{j}\right\|_{q, \alpha}^{q}<\mu_{\alpha}\left(I_{j}\right)$ for each $j$.

The idea will be now to do for each $b_{j}$ the same kind of decomposition that we performed for $b$ (with the same $t$ ) and to bulld an induction process which will eventually lead to the decomposition (3.1). We shall use multi-indices for the successive decomposition, in the following way:

$$
\begin{aligned}
& b(x)=g_{0}(x)+\sum_{j_{0}} h_{j_{0}}(x)=g_{0}(x)+c_{1} t \underset{j_{0}}{\sum} b_{j_{0}}(x) \\
& =g_{0}(x)+c_{1} t \sum_{j_{0}}^{\sum}\left(g_{j_{0}}(x)+\sum_{j_{1}} h_{j_{0}, j_{1}}(x)\right) \\
& =g_{0}(x)+c_{1} t \underset{j_{0}}{\sum} g_{j_{0}}(x)+c_{1} t \underset{j_{0}, j_{1}}{\Sigma} h_{j_{0}, j_{1}}(x) \\
& =g_{0}(x)+c_{1} t \quad \sum_{j_{0}} g_{j_{0}}(x)+\ldots+\left(c_{1} t\right)^{n} \quad \sum_{j_{0}, \ldots, j_{n-1}}^{\sum} g_{j_{0}} \cdots j_{n-1}(x) \\
& +\left(c_{1} t\right)^{n} j_{0}, \ldots, j_{n} h_{0}, \ldots, j_{n}(x)
\end{aligned}
$$

for each $n \in N$, where, $b_{j_{0}, \ldots, j_{n-1}}:=\left(c_{1} t\right)^{-1} h_{j_{0}}, \ldots, j_{n-1}$ and

(i) $\quad \mu_{\alpha}\left(\left\{M_{\alpha}^{q}\left(b_{j_{0}}, \ldots, j_{n-1}\right)>t\right\}<C \mu_{\alpha}\left(I_{j_{0}, \ldots, j_{n-1}}\right) / t^{q}\right.$ 


$$
\begin{aligned}
& \text { (ii) }\left\{M_{\alpha}^{q}\left(b_{j_{0}}, \ldots, j_{n-1}\right)>t\right\}=\bigcup_{j_{n}} I_{j_{0}}, \ldots, j_{n} \\
& \text { (iii) supp } h_{j_{0}}, \ldots, j_{n} \subset I_{j_{0}}, \ldots, j_{n}, \int h_{j_{0}, \ldots, j_{n}} d \mu=0 \\
& \text { (iv) } \left.\frac{1}{\mu_{\alpha}\left(I_{j_{0}}, \ldots, j_{n}\right)} \int_{I_{j_{0}}, \ldots, j_{n}}\left|h_{j_{0}, \ldots, j_{n}}\right|^{q} d \mu_{\alpha}\right)^{1 / q}<C_{1} t, \\
& \text { (v) } \mid g_{j_{0}, \ldots, j_{n-1}} \text { (x) } \mid<c_{0} t,
\end{aligned}
$$

for every $j_{0}, \ldots, j_{n}$ and $n \in N$.

By using (i), (ii) and (iv), we see that the $L_{\alpha}^{1}$-norm of the last term in the right

hand side of (3.3) is bounded by $\left(C t^{1-q}\right)^{n+1} \mu_{\alpha}(I)$. Hence for large $t>0$ so that $\mathrm{Ct}^{1-\mathrm{q}}<1$, we have that

$$
\begin{aligned}
& b(x)=g_{0}(x)+c_{1} t \underset{j_{0}}{\Sigma} g_{j_{0}}(x)+\ldots \\
& +\left(c_{1} t\right)^{n} \quad \sum \quad j_{0}, \ldots, j_{n-1} g_{j_{0}}, \ldots, j_{n-1}(x)+\ldots, \text { in } L_{\alpha}^{1} .
\end{aligned}
$$

Let $a_{0}:=\left(C_{0} t \mu_{\alpha}(I)\right)^{-1} g_{0}$ and $\left.a_{j_{0}}, \ldots, j_{n-1}:=\left(C_{0} t\right) \mu_{\alpha}\left(I_{j_{0}}, \ldots, j_{n-1}\right)\right)^{-1} g_{j_{0}}, \ldots, j_{n-1}$ for

each $j_{0}, \ldots, j_{n-1}, n \in N$, then these are $(1, \infty)_{\alpha}$ atoms by $(1 i 1)$ and $(v)$. Thus we obtain that

$$
\begin{aligned}
a(x) & =\mu_{\alpha}(I)^{-1} b(x) \\
& =C_{0} t \mu_{\alpha}(I)^{-1}\left(\mu_{\alpha}(I) a_{0}(x)+C_{1} t \sum_{j_{0}} \mu_{\alpha} I_{j_{0}}\right) a_{j_{0}}(x)+\ldots \\
& \left.+\left(C_{1} t\right)^{n} \quad \sum j_{0}, \ldots \ldots j_{n-1} \mu_{\alpha}\left(I_{j_{0}}, \ldots, j_{n-1}\right) a_{j_{0}}, \ldots, j_{n-1}(x)+\ldots\right),
\end{aligned}
$$

which is the desired representation (3.1). For, the sum of the absolute value of the coefficients of the right hand side is bounded by $C_{0} t \sum_{0}^{\infty}\left(C t^{1-q}\right)^{k}:=c, c$ independent of a. This completes the proof.

THEOREM C. Let $-1<\alpha<0$ and $1<p_{1}<\infty$. Suppose that $T$ is a sublinear operator of weak-type $(1,1)$ on $H_{\alpha}^{1}$, by which we mean that there exists $B_{0}$ such that for every $f \in H_{\alpha}^{1}$ and $t>0$;

$$
\left.\mu_{\alpha}\{x \quad G ;|\operatorname{Tf}(x)|>t\}\right)<B_{0}|| f \|_{H_{\alpha}^{1}} / t,
$$

and $T$ is of weak-type on $L_{\alpha}^{P 1}$ with constant $B_{1}$. Then for $1<p<p_{1}$, $T$ is of type $(p, p)$ on $L_{\alpha}^{P}$ with constant depending only on $B_{0}, B_{1}, p_{1}$ and $p$.

PROOF. The proof is similar to the non-weighted case [4], [5].

Let $\mathrm{f} \quad \mathrm{L}_{\alpha}^{\mathrm{p}}$ and choose a $\mathrm{q}$ so that $1<\mathrm{q}<\mathrm{p}<\mathrm{p}_{1}<\infty$. As in the proof of Theorem $B$, we consider the open set $E_{t}:=\left\{M_{\alpha}^{q} f>t\right\}=\left\{M_{\alpha}\left(|f|^{q}\right)>t^{q}\right\}$, for $t>0$. 
Then we have the same kind of decomposition; $E_{t}=\bigcup_{j} I_{j}$. From this we obtain a Calderon-Zygnund decomposition $f=g_{t}+h_{t}$, where $g_{t}=f$ if $x \notin E_{t} ;=m\left(f, I_{j}\right)$ if $x \in I_{j}$ for each $j$, and $h:=h_{t}=\sum$, $h_{j}$, where $h_{j}:=\left(f-g_{t}\right) \xi_{I_{j}}$. We then have $\left|g_{t}(x)\right|<C_{0} t$ and

$$
\left(\frac{1}{\mu_{\alpha}\left(I_{j}\right)} \int_{I_{j}}\left|h_{j}\right|^{q} d \mu_{\alpha}\right)^{1 / q}<c_{1} t
$$

for each $j \in N$. Hence $a_{j}:=\left(C_{1} t \mu_{\alpha}\left(I_{j}\right)\right)^{-1} h_{j}$ is $a(1, q)_{\alpha}$ atom and $h=C_{1} t \sum_{j} \mu_{\alpha}\left(I_{j}\right) a_{j} \in H_{\alpha}^{1, q}$. And Theorem B implies that $h \in H_{\alpha}^{1}$ with norm bounded by $C t \mu_{\alpha}\left(E_{t}\right)$. The rest of proof proceed as in [4], [5] with a few modifications, so we omit the details.

4. PROOFS OF THE MAIN RESULTS.

PROOF OF THEOREM 1. Let $-1 / \mathrm{r}^{\prime}<\alpha<0$. To prove the conclusion, it suffices to s show that $\left\|K K_{a}\right\|_{1, \alpha}<c$ for every $(1, \infty)_{\alpha}$ atom a, where $K:=\left(\phi^{k}\right)^{v}$. Let a be such an atom, supported on an interval $I=x_{0}+G_{n}\left(x_{0} \in G, n \in Z\right)$. We write

$$
\int_{G}|K * a| d \mu_{\alpha}=\int+\int_{I}=A+B \text {, say. }
$$

Let first $r=1$ (hence $\alpha=0$ ). Then

$$
A<\left(\int_{I}\left|K^{\star} a(x)\right|^{2} d \mu\right)^{1 / 2}\left(\int_{I} d \mu\right)^{1 / 2}<C|| a||_{2} \mu(I)^{1 / 2}<C \mu(I)^{-1} \mu(I)=C .
$$

On the other hand,

$$
\begin{aligned}
B & \left.\left.=\int_{G I I}\left|\int_{G} K(x-y) a(y) d \mu(y)\right| d \mu(x)=\int_{G} \mid \int_{G} K(x-y)-K\left(x-x_{0}\right)\right) a / y\right) d \mu(y) \mid d \mu(x) \\
< & \int_{I}|a(y)| d \mu(y) \int_{G}\left|K(x-y)-K\left(x-x_{0}\right)\right| d \mu(x) \\
& =\int_{G}\left|a\left(x_{0}+y\right)\right| d \mu(y) \int_{G}|K(x-y)-K(x)| d \mu(x)<C \int_{G}\left|a\left(x_{0}+y\right)\right| d \mu(y)<C .
\end{aligned}
$$

hence the conclusion follows, when $r=1$. This together with Theorem $C$ implies the conclusion of Corollary (ii).

Next, let $r>1$. To estimate $A$, we use Corollary (11) and Lemma (c). Then

$$
\begin{aligned}
A< & \left(\int_{I}\left|K^{* a} a(x)\right|^{r} d \mu\right)^{1 / r}\left(\int_{I}\left(v_{\alpha}(x)\right)^{r^{\prime}} d \mu\right)^{1 / r^{\prime}}<c|| a||_{r}\left(\int_{I} v_{\alpha r^{\prime}}(x) d \mu\right)^{1 / r^{\prime}} \\
& <C \mu_{\alpha}(I)^{-1} \mu(I)^{1 / r} \mu(I)^{1 / r^{\prime}} \inf \left\{v_{\alpha}(x) ; x \in I, x \neq 0\right\}<C_{\alpha}(I)^{-1} \mu_{\alpha}(I)=C .
\end{aligned}
$$

On the other hand, using Lemma (c) again,

$$
B<\int_{I}|a(y)| d \mu(y) \int_{G}\left|K(x-y)-K\left(x-x_{0}\right)\right| v_{\alpha}(x) d \mu(x)
$$




$$
\begin{aligned}
& =\int_{G_{n}}\left|a\left(x_{0}+y\right)\right| d \mu(y) \int_{G G_{n}}|K(x-y)-K(x)| v_{\alpha}\left(x+x_{0}\right) d \mu(x) \\
& =\sum_{\ell=-\infty}^{n-1} \int_{G_{n}}\left|a\left(x_{0}+y\right)\right| d \mu(y) \int_{G_{\ell} \backslash G_{\ell+1}}|K(x-y)-K(x)| v_{\alpha}\left(x+x_{0}\right) d \mu(x) \\
& \left.\sum_{-\infty}^{n-1} \int_{G_{n}}\left|a\left(x_{0}+y\right)\right| d \mu(y) \quad \int_{G_{\ell} \backslash G_{\ell+1}}|K(x-y)-K(x)|^{r} d \mu(x)\right)^{1 / r} \\
& \times\left(\int_{G_{\ell} \backslash G_{\ell+1}} v_{\alpha r^{\prime}}\left(x+x_{0}\right) d \mu(x)\right)^{1 / r^{\prime}} \\
& \left.\sum_{-\infty}^{n-1} \int_{G_{n}}\left|a\left(x_{0}+y\right)\right| d \mu(x)\left(m_{\ell}\right)^{\varepsilon+1 / r^{\prime}}\left(m_{n}\right)^{-\varepsilon} m_{\ell}\right)^{-1 / r^{\prime}} \\
& x \inf \left\{v_{\alpha}(x) ; x \in I, x \neq 0\right\} \\
& <\mathrm{c}\left(m_{n}\right)^{-\varepsilon} \sum_{-\infty}^{\mathrm{n}-1}\left(m_{\ell}\right)^{\varepsilon} \int_{G_{n}}\left|a\left(x_{0}+y\right)\right| v_{\alpha}\left(x_{0}+y\right) d \mu(y)<c\left(m_{n}\right)^{-\varepsilon}\left(m_{n-1}\right)^{\varepsilon}\|a\|_{1, \alpha}<c .
\end{aligned}
$$

This completes the proof.

PROOF OF COROLLARY. (i) Since $\phi \in L^{\infty}(\Gamma)$ is amultiplier on $L^{2}$, it follows from a classical interpolation theorem for weighted spaces [6] and [1, Proposition 1] that $\phi$ is a multiplier on $\mathrm{L}_{\alpha}^{2}$ for all $-\left|\alpha_{0}\right|<\alpha<\left|\alpha_{0}\right|$. As in the proof of 11 , Theorem 1], the case where $1<p<2$ and $-\left|\alpha_{0}\right|<\alpha<0$, has to be proved.

Let $1<p<2$ and $-\left|\alpha_{0}\right|<\alpha<0$. Since each $\phi^{k}, k \in z$ is a multiplier on $L_{\alpha}^{2}$ and also a $\left(H_{\alpha}^{1}, L_{\alpha}^{1}\right)$ multiplier by Theorem 1 , it follows from Theorem $C$ that $\phi^{k}$ is a multiplier on $L_{\alpha}^{p}$. The assumption that the constants $C$ and $\varepsilon$ are independent of $k$, implies that $\phi$ is a multiplier on $L_{\alpha}^{p}$.

(1i) This is already seen in the proof of Theorem 1 .

PROOF OF THEOREM 2. According to Theorem A, it suffices to show that $\left\|\left(\check{\sim}^{*}\right)_{a}^{*}\right\|_{1, \alpha}<\mathrm{C}$ for all $(1, \infty)_{\alpha}$ atom a. Let a be a $(1, \infty)_{\alpha}$ atom, supported on an interval $I:=x_{0}+G_{n}\left(x_{0} \in G, n \in Z\right)$. We set $\check{\phi}^{*} a=f$. The case where $r=1$ (hence $\alpha=0$ ) is known [2, corollary]. So we let $1<r<\infty$ and $-1 / r^{\prime}<\alpha<0$. Now we write

$$
\int_{G} f * d \mu_{\alpha}=\int_{I}+\int_{G \backslash I}=A+B, \text { say. }
$$

We first estimate A. Since $k_{r}^{1 / r^{\prime}+\varepsilon, \infty} \quad k_{1}^{\varepsilon, \infty}$ Lemma (b) and [2, Corollary]

imply that $\left\|\mathrm{f}^{*}\right\|_{\mathrm{r}}<\mathrm{c}\|\mathrm{f}\|_{\mathrm{r}} \leqslant \mathrm{c}\|\mathrm{a}\|_{\mathrm{r}}$. Thus as in the proof of Theorem 1 , we have that

$$
\begin{gathered}
A<\left(\int_{I}\left(f^{*}\right)^{r} d \mu\right)^{1 / r}\left(\int_{I} v_{\alpha r^{\prime}} d \mu\right)^{1 / r^{\prime}}<C\|a\| \mu(I)^{1 / r^{\prime}} \inf \left\{v_{\alpha}(x) ; x \in I, x \neq 0\right\} \\
<C \mu_{\alpha}(I)^{-1} \mu_{\alpha}(I)=C
\end{gathered}
$$


Let $\psi(\gamma):=\overline{\left(\gamma, x_{0}\right)} \phi(\gamma)$ and $b(x):=a\left(x+x_{0}\right)$. Then it is easily seen that $f=\phi \star_{a}=\psi \star_{b}$, supp $b \subset G_{n}$, and $\int b d \mu=0$. Thus we have that $b * D_{k}=0$ if $k<n$, and supp $\left(b * D_{k}\right) \subset G_{n}$ if $k>n$. Also $\left(b * D_{k}\right)_{j}:$ $=\left(b * D_{k}\right) *\left(D_{j+1}-D_{j}\right)=0$ if $j>k$ and $\left(b * D_{k}\right)_{j}=b_{j}$ if $j<k$. Moreover $b_{j}=0$, if $j<n$. Hence

$$
\begin{aligned}
& \left.f^{*}(x)=(\check{\psi} * b) *=\sup _{k} \mid \stackrel{\sim}{(\psi * b}\right) * D_{k}(x)\left|\quad=\sup _{k>n}\right| \check{\psi} *\left(b * D_{k}\right)(x) \mid
\end{aligned}
$$

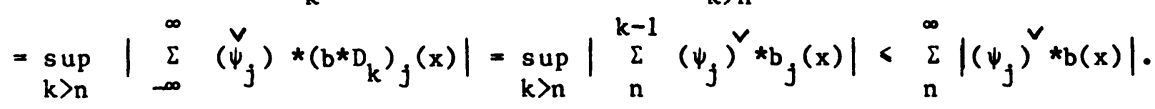

Then,

$$
B=\int_{G \backslash I} f^{\star} d \mu_{\alpha}<\sum_{n}^{\infty} \int_{G \backslash I}\left|\left(\psi_{j}\right)^{v} \star b\right| d \mu_{\alpha}=\sum_{j=n}^{\infty} \sum_{i=-\infty}^{n-1} \int_{i} \int_{i+1}\left|\left(\psi_{j}\right) \star b\right| d \mu_{\alpha},
$$

where $I_{i}:=x_{0}+G_{1}$ for each $i \in Z$.

Now for $i<n$,

$$
\begin{aligned}
& \left(\dot{\psi}_{j}\right)^{\vee} \star_{b}(x)=\int_{G}\left(\psi_{j}\right)^{\vee}(y) b(x-y) d \mu(y) \\
& =\int_{I_{i+1}}+\int_{I_{i} \backslash I_{i+1}}+\int_{G \backslash I_{i}}
\end{aligned}
$$

If $x \in I_{i} \backslash I_{i+1}$ and $y \in I_{i+1}, x-y \in G_{i} \backslash G_{i+1} \subset G G_{n}$. Also

if $x \in I_{i} I_{i+1}$ and $y t I_{i}, x-y \in G \backslash G_{i} \subset G \backslash G_{n}$. These, together with supp $b C G_{n}$ imply that the first and last terms of the right hand side of the equality are zero. Thus (4.2) is bounded by

$$
\begin{aligned}
& \sum_{j=n}^{\infty} \sum_{i=-\infty}^{n-1} \int_{J_{i}}\left|\left(\left(\psi_{j}\right)^{v} \xi_{J}\right) * b\right| d_{1} \mu_{\alpha} \\
& \left.<\sum_{n}^{\infty} \sum_{-\infty}^{n-1} \int_{G}|b(y)| d \mu(y) \int_{J_{1}} \mid\left(\psi_{j}\right) \xi_{J_{i}}\right)(x-y) \mid d \mu_{\alpha}(x)
\end{aligned}
$$

where $J_{i}:=I_{i} \backslash I_{i+1}$ for each $i \in Z$. Now, Lemma (c)

$$
\begin{aligned}
& \int_{J_{i}}\left|\left(\left(\psi_{j}\right)^{v} \xi_{J_{i}}\right)(x-y)\right| d \mu_{\alpha}(x) \\
& <\left(\int_{J_{i}}\left|\left(\left(\psi_{j}\right)^{V_{J_{j}}}\right)(x-y)\right|^{r} \mathrm{~d}_{\mu}(x)\right)^{1 / \mathrm{r}}\left(\int_{I_{i}} v_{\alpha r^{\prime}}(x) d \mu(x)\right)^{1 / r^{\prime}} \\
& <\left(\int_{J_{i}} \mid\left(\left.\left(\psi_{j}\right)^{V}(x)\right|^{r} d_{\mu}(x)\right)^{1 / r}\left(m_{i}\right)^{-1 / r^{\prime}} \inf \left\{v_{\alpha}(x) ; x \in I_{i}, x \neq 0\right\}\right. \\
& =\left(\int_{G_{i} \backslash G_{i+1}}\left|\left(\phi_{j}\right)^{v}(x)\right|^{r} d_{\mu}(x)\right)^{1 / r}\left(n_{i}\right)^{-1 / r^{\prime}} \inf \left\{v_{\alpha}(x) ; x \in I_{i}, x \neq 0\right\}
\end{aligned}
$$


Hence $(4.3)$ is bounded by

$$
\begin{aligned}
<\sum_{j=n}^{\infty} \sum_{i=-\infty}^{i-1} \int_{G}\left|a\left(y+x_{0}\right)\right| d \mu(y) \dot{i} a f\left\{v_{\alpha}(x) ; x \in I_{i}, x \neq 0\right\} \\
\quad x\left(m_{i}\right)^{-1 / r^{\prime}}\left(\int_{G_{i} \backslash_{i+1}}\left|\left(\phi_{j}\right)^{V}(x)\right|^{r} d \mu(x)\right)^{1 / r}
\end{aligned}
$$

Since $I=I_{n} \subset I_{1}(i<n)$ and $\|a\|_{1, \alpha}<1$,

$$
\begin{aligned}
& B \leqslant C \quad \sum_{j=11}^{\infty} \sum_{i=-\infty}^{n-1}\left(m_{i}\right)^{-1 / r^{\prime}}\left(\int_{I}|a(y)| d_{\mu}(y) \text { inf }\left\{v_{\alpha}(x) ; x \in I, x \neq 0\right\}\right. \\
& \times\left(\int_{G_{i} \backslash G_{i+1}}\left|\left(\phi_{j}\right)^{v}(x)\right|^{r} d_{\mu}(x)\right)^{1 / r} \\
& <C \sum_{n}^{\infty} \sum_{-\infty}^{n-1} m_{i}^{\varepsilon}\left(m_{i}\right)^{-\left(1 / r^{\prime}+\varepsilon\right)}\left(\int_{G_{i} G_{i+1}}\left|\left(\phi_{j}\right)^{v}\right|^{r} d_{\mu}\right)^{1 / r} \\
& \leqslant C \sum_{n}^{\infty} \sum_{-\infty}^{n-1} m_{i}^{\varepsilon}\left\|\left(\phi_{j}\right)\right\|_{K_{r}^{\varepsilon}+1 / r^{\prime}, \infty}<c \sum_{n}^{\infty}\left(m_{j}\right)^{-\varepsilon} \sum_{-\infty}^{n-1} 10_{i}^{\varepsilon}<C . \quad(4.4)
\end{aligned}
$$

Hence, we have that $\left\|f^{*}\right\|_{1, \alpha} \leqslant c$ by $(4.1)$ and (4.4). This completes the proof.

\section{REFERENCES}

1. ONNEWEER, C.W. Multipliers on weighted $\mathrm{L}^{\mathrm{P}}$-spaces over certain totally disconnected groups, Trans. Amer. Math. Soc. 288 (1985), 347-362.

2. KITADA, T. $\mathrm{H}^{\mathrm{P}}$-multiplier theorems on certain totally disconnected groups, Sc1 Rep. Hirosaki Univ. 34 (1987), 1-7.

3. ONNEWEER, C.W. Generalized Lipshitz spaces and Herz spaces on certain totally disonneted groups, Lec. Notes in Math., no. 939, Springer 1982, 106-121.

4. COIFMAN, R.R. and WEISS, G. Extensions of Hardy spaces and their use in analysis, Bul1. Amer. Math. Soc. 83 (1977), 569-645.

5. GARCIA-CUERVA, J. and DE FRANCIA, J.L. RUBIO. Weighted norm inequalities and related topics, Mathematics Studies 116, North-Holland (1985).

6. STEIN, E.M. Interpolation of linear operators, Trans. Amer. Math. Soc. 83 (1956), 482-492. 


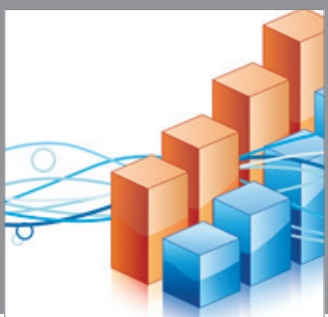

Advances in

Operations Research

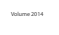

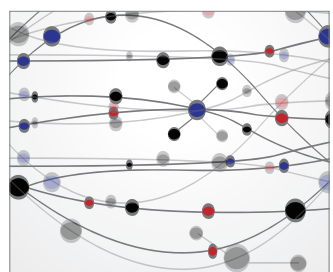

\section{The Scientific} World Journal
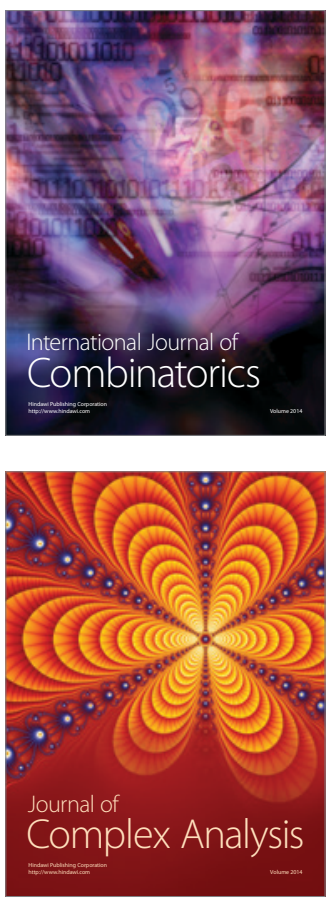

International Journal of

Mathematics and

Mathematical

Sciences
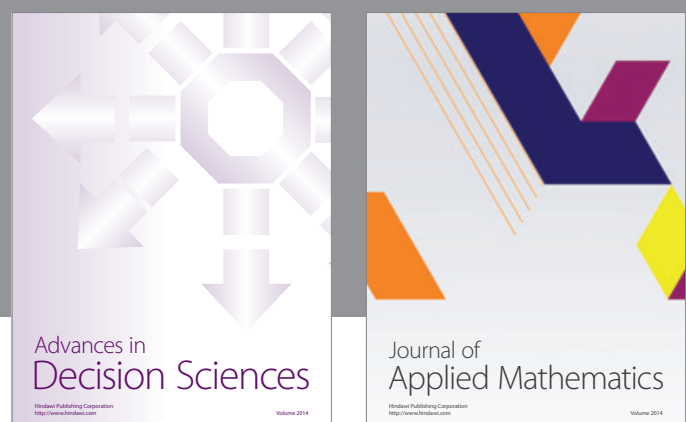

Journal of

Applied Mathematics
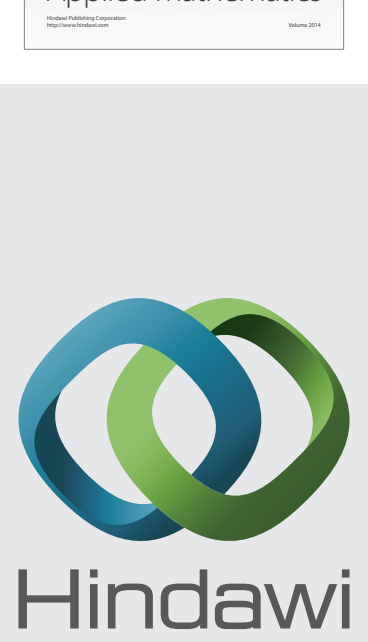

Submit your manuscripts at http://www.hindawi.com
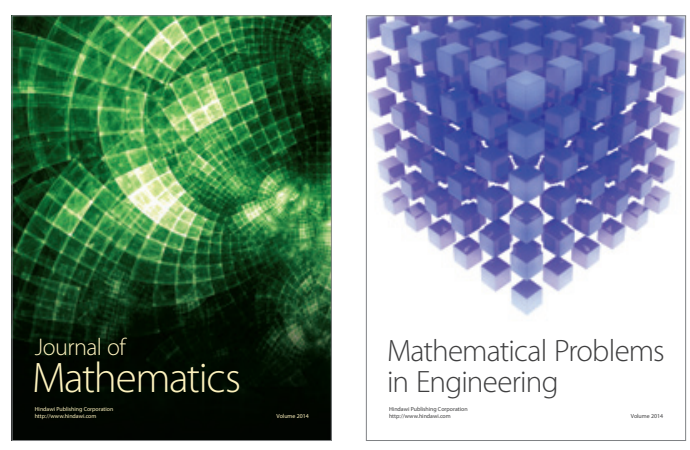

Mathematical Problems in Engineering
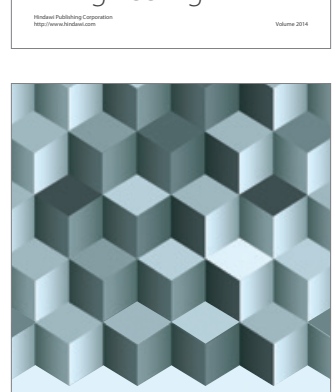

Journal of

Function Spaces
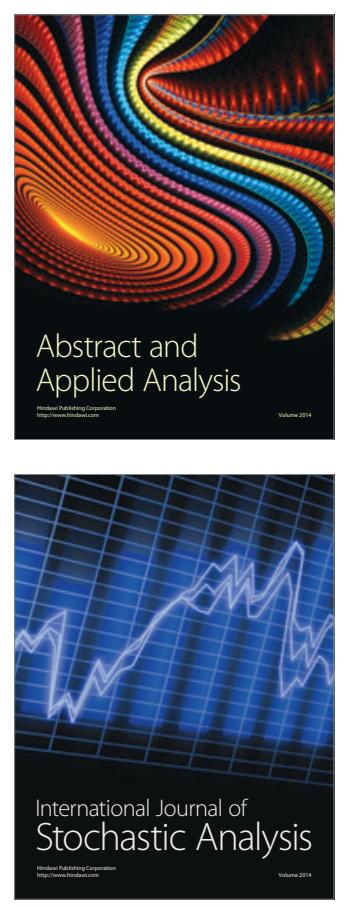

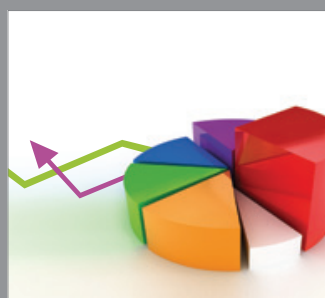

ournal of

Probability and Statistics

Promensencen
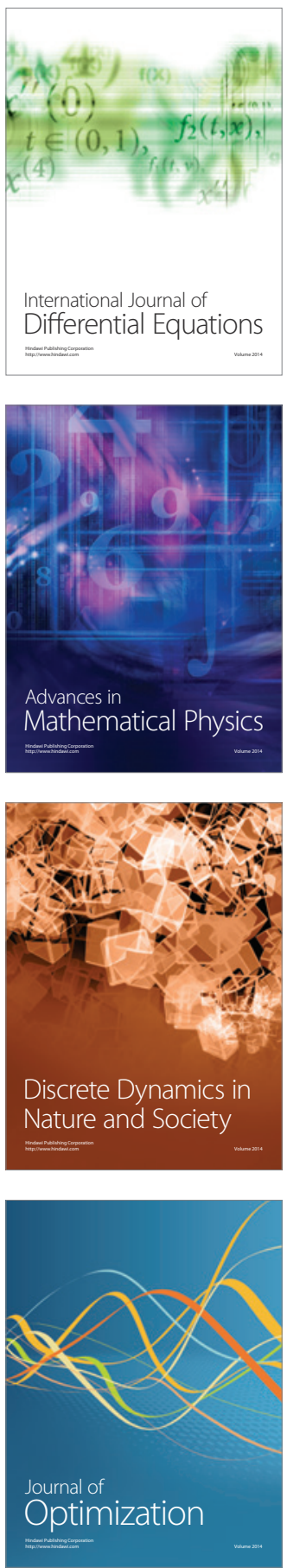